\title{
Recuento de dos poblaciones de especies de anfibios Rhinella spinulosa y Telmatobius jelskii, en el Valle del Mantaro
}

\section{Recounting two populations of amphibian species, Rhinella spinulosa and Telmatobius jelskii in the Mantaro Valley}

\author{
Verónica Canales Guerra \\ Universidad Continental \\ vcanales@continental.edu.pe
}

\section{RESUMEN}

El objetivo de este trabajo de investigación fue estudiar dos poblaciones de anfibios representativos de los dos ambientes donde estos vertebrados se encuentran Rhinella spinulosa de hábitos terrestres y Telmatobius jelskii de hábitos acuáticos en los ríos Shullcas, Chanchas y Cunas, en la provincia de Huancayo, departamento de Junín a una altitud de $3200 \mathrm{msnm}$, con el fin de determinar la distribución actual de ambas especies. Se utilizó el método de Registro de Especies por Encuentro Visual REV. Respecto a los resultados de este estudios podemos mencionar que en el río Shullcas se encontraron solo dos individuos juveniles de metamorfosis completa de la especie Rhinella spinulosa; no se encontró ningún individuo de la especie Telmatobius jelskii. En el río Chanchas se encontró una población de anfibios de la especie $R$. spinulosa en las tres réplicas de muestreo, se capturaron algunos individuos para la toma de datos, y todos eran de aproximadamente la misma edad, juveniles de pequeño tamaño que habían terminado recientemente la metamorfosis y no se encontraron individuos de la especie $T$. jelskii. En el río Cunas solo se halló 7 individuos de la especie $R$. spinulosa en las tres procesos de recolección los cuales se ubicaron en forma aislada en diversos puntos. En los hábitats investigados se observaron alteraciones antrópicas, siendo la más notoria, el cambio del uso de tierras. En los tres ríos estudiados se encontró alteración de los hábitats lo cual ha modificado claramente la distribución y abundancia de las poblaciones esto podría ser la principal causa de la desaparición de las poblaciones.

\section{ABSTRACT}

The aim of this research was to study two representative amphibians' populations; Rhinella spinulosa of terrestrial habits and Telmatobius jelskii of aquatic ones; to determine the current distribution of both species in the Shullcas, Chanchas and Cunas rivers in the Huancayo province, department of Junín, located at 3200 meters of altitude. We used the Species Registration method by Visual Encounter (REV). Regarding the results of this study there were only two juvenile individuals of complete metamorphosis belonging to the species Rhinella spinulosa in the Shullcas River; no individual of the species Telmatobius jelskii was found. An amphibian population of the species $R$. spinulosa was found in the Chanchas River in three sampling replicates; some individuals of about the same age, small juveniles who had recently finished metamorphosis, were captured for data collection; and no individuals of the species T. jelskii were found. In the Cunas River only 7 individuals of the R. spinulosa species were found in the three collecting processes which were placed in isolation in several points. In the investigated habitats anthropogenic disturbances were observed being the most notorious, a changing in land use. Habitat alteration was found in the three rivers studied, which clearly modified the distribution and abundance; this could be the main cause of the populations' disappearance.

Keywords: Amphibian, mantaro valley, telmatobius jelskii, rhinella spinulosa.

Palabras clave: Anfibios, Valle del Mantaro, Telmatobius jelskii, Rhinella spinulosa.

Historial del artículo:

Recibido: 14 de noviembre de 2014. Aprobado: 23 de febrero 2015. Disponible en línea: 30 de junio de 2015

1 Docente de la Universidad Continental y responsable del curso de Biología. 


\section{INTRODUCCIÓN}

Los anfibios enfrentan en la actualidad, una condición que es el resultado de una sinergia de varias amenazas que atentan contra la supervivencia de estos vertebrados $(1,2,3)$, en todo el mundo y el Perú al ser un país también en este aspecto (como en la amplia biodiversidad) muy diverso no es ajeno a esta problemática.

El objetivo de este estudio es hacer una evaluación de la distribución de las poblaciones de dos especies de anfibios conocidos en la zona de la ciudad de Huancayo departamento de Junín, de las cuales no existe registro reciente. Hasta unos 20 años atrás, estas dos especies habitaron en todo el Valle del Mantaro $(4,5)$. Sin embargo debido a que la distribución de tierras en la ciudad está cambiando rápidamente, se hace necesario una evaluación que permita conocer

\section{MATERIAL Y MÉTODOS}

\section{Área de estudio}

El muestreo se realizó en el Valle del Mantaro, Andes Centrales a 3200 msnm en la ciudad de Huancayo, departamento de Junín, en un área delimitada por los ríos: Cunas al noroeste, Shullcas al este y Chanchas al sur, durante el período setiembre - diciembre del 2013, coincidiendo con el inicio de la estación de Iluvias en esta región. Estos tres ríos delimitan el área urbana del valle y es la zona donde se realizan la mayor cantidad de cambios en el uso de tierras que fueron antes agrícolas y ahora residenciales $\mathrm{u}$ otros. (Figura 1).

El tramo del río Cunas estudiado se encuentra en el distrito de Pilcomayo y en la actualidad se extraen

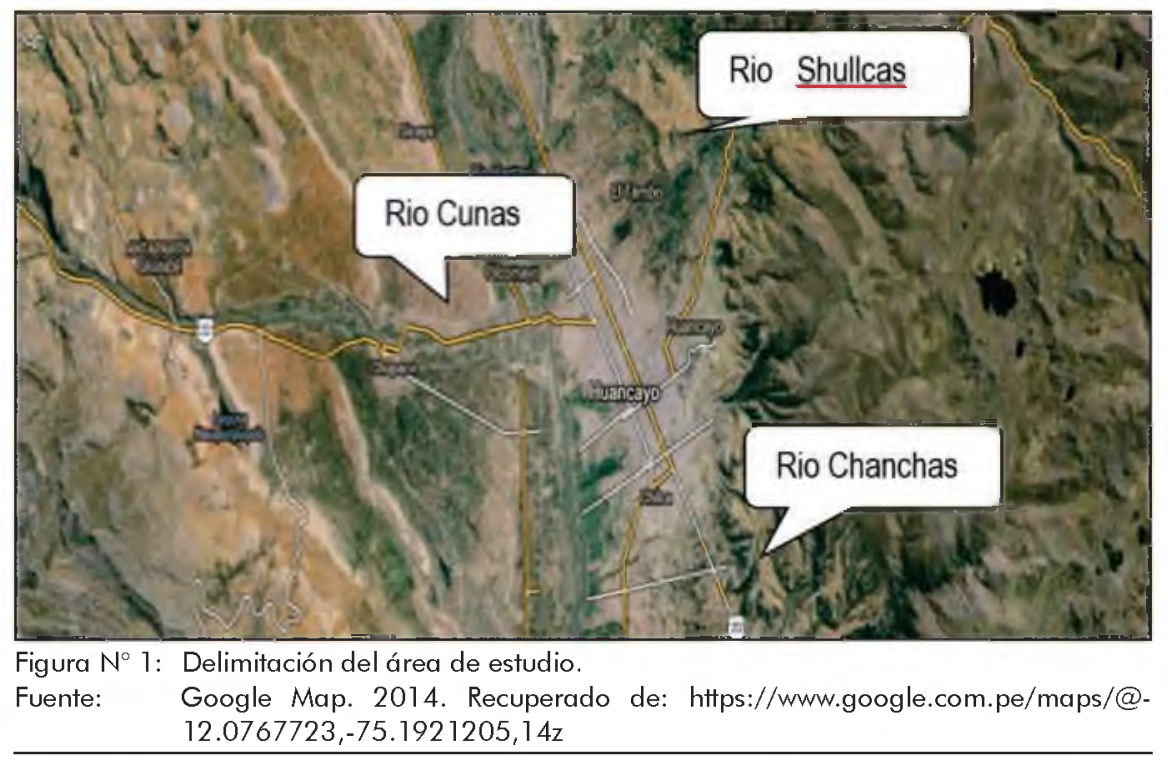

el estado actual de ambas poblaciones para probar si este cambio ha afectado los hábitats de las especies.

El área de estudio está delimitada naturalmente por el curso de tres ríos: Chanchas, Shullcas y Cunas, que a su vez limitan el área urbana de la ciudad. Las poblaciones estudiadas son Rhinella spinulosa, una especie preferentemente terrestre, que habita las proximidades de ríos y riachuelos, aunque los adultos pueden encontrarse en los terrenos aledaños, y la especie Telmatobius jelskii que habita ríos y riachuelos durante todo su ciclo biológico $(6,7)$.

No se encuentran datos recientes de las poblaciones estudiadas en la bibliografía consultada, sin embargo se sabe que la mayoría de especies del género Telmatobius se encuentran en franco declive (8). piedras para ser utilizadas en la construcción, por ello la ribera del río no se halla vegetación. Las áreas verdes a los lados oscilan entre $2 \mathrm{~m}$ y $5 \mathrm{~m}$, luego encontramos chacras con diversos sembríos o construcciones de viviendas semirurales. El muestreo se realizó $550 \mathrm{~m}$ rio arriba y hasta la desembocadura con el Rio Mantaro $(9,10)$. El tramo estudiado del río Shullcas atraviesa la ciudad de Huancayo, últimamente los bordes están siendo modificados con rocas atadas con mallas de alambre para evitar las inundaciones en la época de lluvia. El límite urbano ha avanzado rápidamente en los últimos 5 años, encontrándose casas a $5 \mathrm{~m}$ del rio, el hábitat de la fauna antes presente ha sido modificado rápidamente. El río fue recorrido desde la localidad de Palian hasta su desembocadura con el Rio Mantaro $(9,10)$. El tramo del Rio Chanchas que se estudió, se encuentra al sur de la ciudad en el distrito de Sapallanga. Es una zona menos urbana, aun 
rodeada de chacras con cultivos diversos, sin embargo encontramos que en los bordes del río aún se estila lavar la ropa con detergente. Para su evaluación fue recorrido desde $500 \mathrm{~m}$ rio arriba del Puente Cunas hasta la desembocadura en el río Mantaro $(9,10)$.

\section{Técnica de muestreo}

La técnica de muestreo elegida para este trabajo descriptivo simple, pertenece a un grupo de técnicas estandarizadas que se detallan en Heyer, et al. $(9,10)$. Se escogieron estas técnicas sólo porque son flexibles y pueden adaptarse a la topografía y apoyo logístico de cualquier área y nos permitirá conocer el estado actual de las especies estudiadas.

En la técnica de Registro de Especies por Encuentro Visual REV, se trata de recorrer transectos previamente determinados en el área de estudio y hacer registros con el fin de poder hacer réplicas del trabajo en fases posteriores. Se trazaron transectos terrestres para el monitoreo de Rhinella spinulosa, de quien no se hizo el registro de la vocalización debido a que no se encontraron suficientes adultos en fase reproductiva. A lo largo de los bordes de los ríos, entre las piedras se buscó a la especie Telmatonius jelskii, de hábitat totalmente acuático. De esta manera se abarcaron los dos hábitats representativos de los anfibios en estudio y también dos ambientes característicos para analizar: suelo y agua.

El método consiste en que dos o más personas caminan lentamente a lo largo del transecto previamente trazado y cuidadosamente buscan sapos sobre el suelo. Una persona hace las observaciones mientras que la otra registra toda la información. La distancia efectiva de cada transecto fue de aproximadamente de 1 a 3 metros a cada lado de la ribera del rio, dependiendo de la densidad de la vegetación y el relieve. En los tres ríos monitoreados, los espacios después de los $3 \mathrm{~m}$ eran chacras sembradas, en el río Shullcas además encontramos cercos vivos de Eucaliptos de $5 \mathrm{~m}$ de alto.

Se identificaron las especies en el lugar y momento en que se localizaron, por medio de una clave para determinación de anfibios de las especies estudiadas, (Anfibios de la sierra central del Perú: una clave de identificación para adultos y larvas (5). Se capturaron los sapos para determinar además de la especie el sexo y edad (adulto, juvenil), también se midieron la longitud y peso de cada individuo, se registró información básica sobre lo que estaba haciendo (actividad) y el lugar donde se encontró (sustrato). Luego se registraró mediante GPS Garming 86, las coordenadas de la localización de cada individuo y la hora de captura. El animal se manipuló lo menos posible y se liberó rápidamente en el mismo lugar donde se capturó. Los datos fueron registrados en tablas Ilamadas Datos de Campo $(9,10)$.

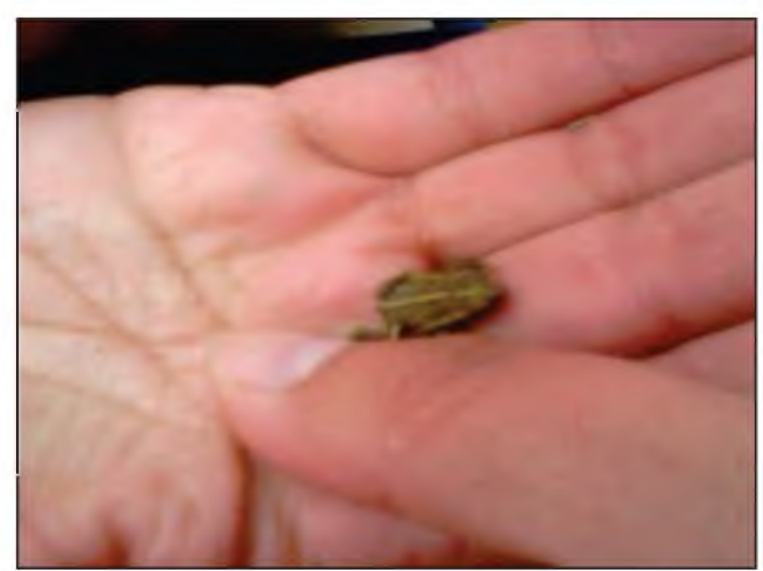

Figura $N^{\circ} 2$ : R. spinulosa juvenil encontrada en el Rio Chanchas.

Los trabajos de muestreo de campo fueron realizados por un equipo que incluye al grupo de Proyección Social de la Universidad Continental: Indicadores Ambientales, los cuales fueron capacitados en tres fechas diferentes en relación a los objetivos de la investigación, la técnica de muestreo y el manejo de los instrumentos de campo y se les entregó una guía de información para obtener los datos de campo. Se realizaron en total 10 salidas de campo entre setiembre y diciembre del 2013.

\section{Telmatobius jelskii}

El género Telmatobius se halla presente en los ríos de las montañas de los Andes o extra-andinos, desde Ecuador hasta la mitad de Argentina y Chile. Muestran un declive de las poblaciones a nivel mundial ampliamente registrado $(1,2)$, debido a que presentan características que los hacen particularmente susceptibles: ciclo de vida totalmente acuático, baja tasa de fecundidad, presente en ríos de altura y distribución endémica (6). Los adultos tienen hábitats escondidos debajo de las rocas dentro de los ríos o forman cavidades en las paredes de los bordes donde se sumergen en la vegetación. En las noches estas ranas se tornan más activas saliendo de sus refugios en busca de alimento (5). En el Perú contamos con 25 especies de estos anfibios, 21 de las cuales se encuentran en distintas categorías de amenaza siendo las principales causas la pérdida del hábitat y la sobre explotación para el consumo (1).

Aunque sólo es evidencia circunstancial la depredación de los renacuajos de Telmatobius por la trucha, la introducción de este pez exótico podría ser otra de las amenazas para las ranas andinas acuáticas. Estudios sobre el declive de Telmatobius en Ecuador y Argentina sugieren que la introducción de especies exóticas de salmónidos (comúnmente conocidas como truchas) puede ser una de las causas de su declive (6). En nuestra región la presencia de estas ranas acuáticas es 
histórica, siendo incluso parte de nuestras costumbres como fuente de alimento (caldos, jugos, extractos) y hasta se le atribuyen cualidades curativas.

\section{Rhinella spinulosa}

Especie de tamaño mediano o grande, entre 45 y 110 $\mathrm{mm}$. Tiene el cuerpo robusto, la cabeza más ancha que larga, los ojos grandes y prominentes y la piel muy glandular. Los machos son de color amarillento a gris verdoso y tienen cornificaciones en forma de espinas. Las hembras son de color café grisáceo y tienen la piel lisa, la mayoría con manchas oscuras en el vientre. Es una especie de hábitos principalmente terrestres, habitando cerca de ríos, vertientes, lagunas y vegas de altura. Los huevos son puestos en cintas de miles de huevos de color negruzco rodeados de material gelatinoso, que se depositan en las orillas de ríos, lagunas y vegas. Las larvas son de color negro y miden hasta $35 \mathrm{~mm}$. Se alimentan principalmente de artrópodos. Se distribuye en la cordillera de Los Andes desde los $9^{\circ} \mathrm{S}$ hasta los $46^{\circ} \mathrm{S}$, en Perú, Bolivia, Chile y Argentina; desde los 1200 msnm en la zona centrosur de Chile hasta los $4600 \mathrm{msnm}$ en el extremo norte (lago Chungará).

Esta especie es abundantemente presente desde años anteriores de acuerdo a lo registrado en el trabajo de $U$. Sinsch (5), y no hay registros posteriores de las especies en la literatura.

Actualmente se encuentra en una categoría de riesgo menor, con poblaciones grandes (11).

\section{RESULTADOS}

\section{Río Shullcas}

En el total de salidas realizadas se encontraron solo dos individuos juveniles de metamorfosis completa, de la especie R. spinulosa (Figura 2). El río mostraba recientes pasos de maquinaria pesada y se notaba que los bordes habían sido modificados con la construcción de barreras para evitar inundaciones (Figura 1). Los individuos encontrados se hallaban en el borde del río cerca de un campo donde jugaban futbol. El río estaba cargado medianamente de acuerdo a la iniciada temporada de lluvia. No se encontró ningún individuo de la especie Telmatobius jelskii.

\section{Río Chanchas}

El río se encontraba cargado y presentaba vegetación a ambos lados. En un punto de suelo bastante húmedo, se encontró una población de anfibios de la especie R. spinulosa en las tres réplicas de muestreo (Figura 3). Se capturaron algunos individuos para la toma de datos, y todos eran de aproximadamente la misma edad, juveniles de pequeño tamaño que habían terminado recientemente la metamorfosis. (Figura 4). En los muestreos dentro del río se encontraron alevinos de truchas pero no se encontraron individuos de la especie T. jelskii.

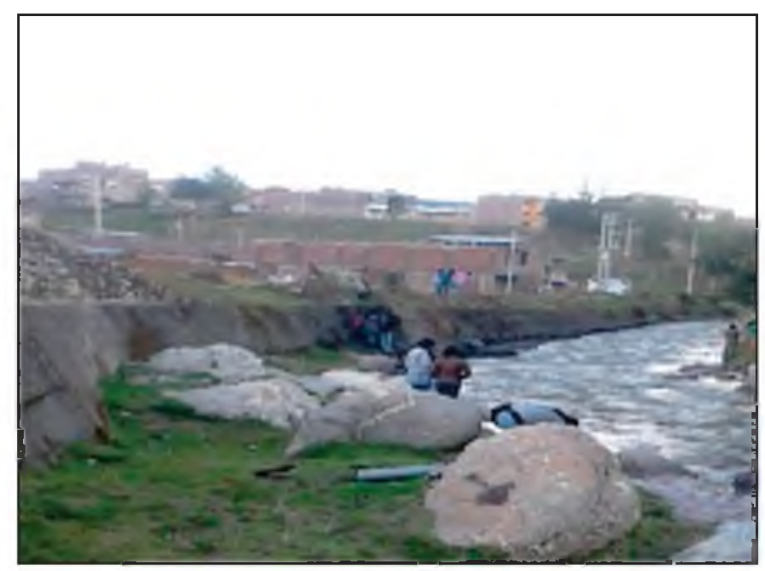

Figura $N^{\circ} 3$ : Rio Shullcas: Bordes con barreras para las inundaciones.

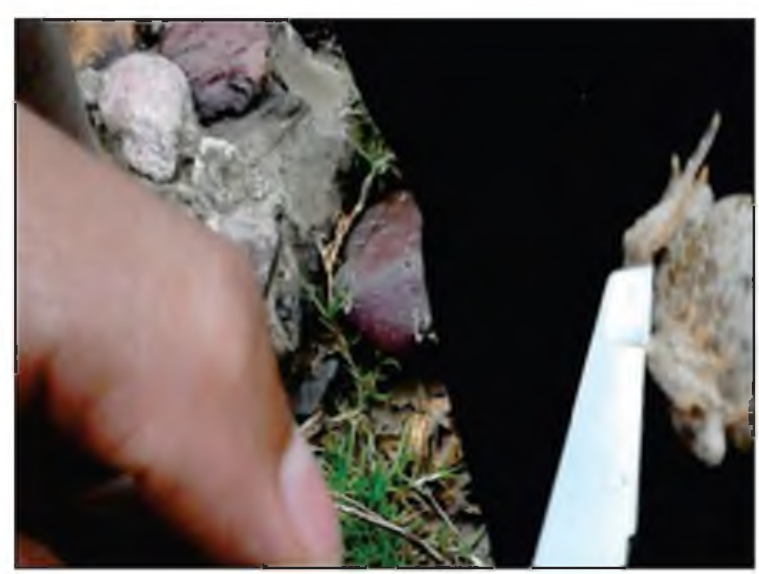

Figura $\mathrm{N}^{\circ} 4$ : Individuo encontrado.

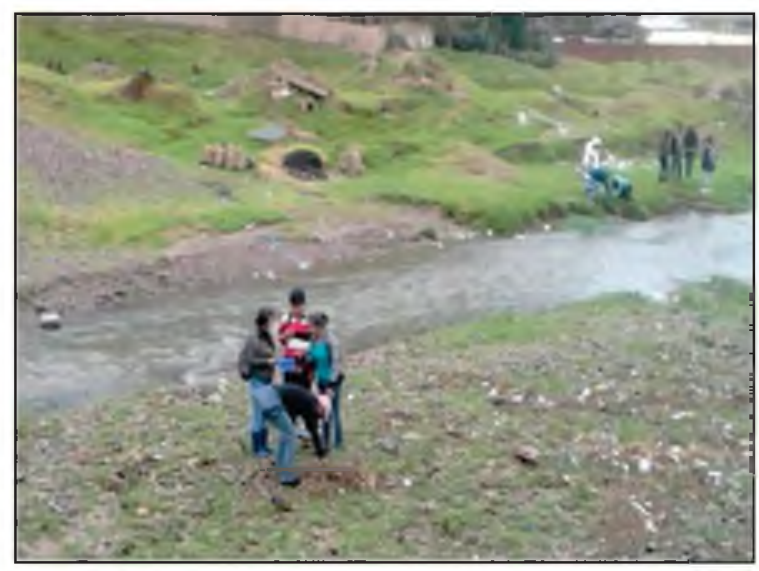

Figura $N^{\circ}$ 5: Río Chanchas. Lugar donde se encontró la población de R. spinulosas. 
Muy cerca del lugar donde se encontró la población, también se encontraron pozas cavadas en el piso, con evidencia de haber sido utilizadas recientemente para el lavado de ropa, como se ve en la Figura 6.

\section{Río Cunas}

El río también se encontraba crecido como en los anteriores casos. La particularidad aquí fue encontrar las riberas con señales de actividad de extracción de material para la construcción. El total de individuos hallados fue de 7 en las tres salidas realizadas, los cuales se ubicaron en forma aislada en los puntos que señala el siguiente mapa que además muestra los lugares de extracción de material.

\section{DISCUSIÓN}

Los antecedentes históricos y bibliográficos, nos dicen que en la zona de estudio era común encontrar poblaciones de anfibios de ambas especies estudiadas, sin embargo las observaciones previas al estudio mostraban lo contrario. Esto fue comprobado por el estudio realizado ya que no se encontraron poblaciones definidas, sólo algunos individuos aislados, excepto en el río Chanchas donde se ubicó un punto que tenía una población abundante de R. spinulosa. (Tabla 1). Sería importante seguir el desarrollo de dicha población y proteger su desarrollo.

Por lo menos en el área de estudio, no se encontraron poblaciones de T. jelskii, que además constituye una especie vulnerable a la contaminación y a los cambios en su hábitat, lo cual estaría de acuerdo a la reducción de las poblaciones de estos anfibios registradas para todo el país por C. Aguilar (1).

Algunas conclusiones son: En la zona de estudio no se encontraron poblaciones de la especie Telmatobius jelskii, sin embargo fueron notables las alteraciones causadas por intervención humana, y la presencia de alevinos de trucha, predadores de la especie estudiada. Se ubicó solamente una población definida de la especie R. spinulosa en el Rio Chanchas en la zona sur de la ciudad y debido a que en la misma zona se hallaron pozas de lavado conteniendo restos de detergente, se hace evidente que la población se halla en peligro.

Ya que en los tres ríos estudiados encontramos alteración de los hábitats lo cual ha modificado claramente la distribución y abundancia de las poblaciones podríamos decir que esto es la principal causa de la desaparición de las poblaciones antes registradas.

El presente trabajo estuvo bastante limitado en tiempo y área de estudio para concluir que no existe ya la especie T. jelskii, sin embargo esta información tiene trascendencia a nivel nacional, ya que otras especies del mismo género presentan el mismo peligro según la información del International Union for Conservation of Nature, IUCN (11).

\section{Agradecimientos}

A los alumnos de la E.A.P. Ingeniería Ambiental $2^{\circ}$ y $4^{\circ}$ "Indicadores Ambientales", que colaboraron en las salidas de campo.

\section{REFERENCIAS BIBLIOGRÁFICAS}

1. Aguilar C, Ramírez C, Rivera D, Siu-Ting K, Suarez J, Torres C. Anfibios andinos del Perú fuera de Áreas Naturales Protegidas: amenazas y estado de conservación. biol. 2010; 17(1): 5-28.

2. Stuart SN, Chanson JS, Cox NA, Young BE, Rodrigues AS, et al. Status and trends of amphibian declines and extinctions worldwide. Science. 2004; 306: 1783-1786.

3. Young BE, Stuart SN, Chanson JS, Cox NA, Boucher TM. Disappearing Jewels: The Status of NewWorld Amphibians. Arlington: NatureServe; 2004.

4. Sinsch U. El Sapo Andino Bufo spinulosus: Análisis Preliminar de su orientación hacia sus lugares de reproducción. Boletín de Lima. 1988; 10(57): 8391.

5. Sinsch U. Anfibios de la sierra central del Perú. Una clave de identificación para adultos y larvas. Boletín de Lima. 1986; 8(45): 23-33.

6. Duellman WE, Trueb L. Biology of Amphibians. Nueva York: McGraw-Hill; 1986.

7. Sinsch U, Salas A, Canales V. Reassessment of central Peruvian Telmatobiinae (genera Batuachophrynus and Telmatobiusl) Morphometry and classification). Alytes. 1995; 13(1): 14-44.

8. Barrionuevo S, Ponssa ML. Decline of three species of the genus Telmatobius (anura: leptodactylidae) from Tucumán Province, Argentina. Herpetologica. 2008; 64(1): 47-62.

9. Angulo A, Rueda-Almonacid JV RodríguezMahecha, La Marca JV. Técnicas de inventario y monitoreo para los anfibios de la región tropical andina. Bogotá D.C: Conservación Internacional; 2006.

10. Lips K, Reaser J, Young B. El Monitoreo de Anfibios en América Latina: Manual para coordinar esfuerzos. The Nature Conservancy; 1999.

11. International Union for Conservation of Nature [Internet]. Gland: IUCN; [Citado el 20 de enero de 2015]. Conservation tools [ 1 pantalla]. Disponible en: http://www.iucn.org/ 